\title{
SUSPENSIÓN TRANSVAGINAL ALTA A LIGAMENTOS UTEROSA- CROS PARA EL TRATAMIENTO DE DEFECTOS APICALES (STALUS): DESCRIPCIÓN DE LA TÉCNICA Y RESULTADOS ANATÓMICOS A MEDIANO PLAZO
}

Jorge Lecannelier A. 1,2, César Sandoval S. 1,2, Michel Naser N. ${ }^{1}$, Valentín Manríquez G. ${ }^{1}$, Rodrigo Guzmán R. ${ }^{1}$, Raúl Valdebenito S. ${ }^{1}$, Gabriel Cavada C. ${ }^{3}$, Amalia Aguilera M. ${ }^{1 a}$, Xi Rao. ${ }^{b}$

1 Unidad de Piso Pélvico Femenino, Departamento de Obstetricia y Ginecología, Hospital Clínico, Universidad de Chile.

2 Unidad de Uroginecología, Departamento de Obstetricia y Ginecología, Clínica Alemana. ${ }^{3}$ Escuela de Salud Pública, Unidad de Bioestadística, Facultad de Medicina, Universidad de Chile.

$a_{\text {Matrona. }}{ }^{b}$ Interna, Escuela de Medicina, Universidad de Chile.

\section{RESUMEN}

Objetivos: Describir una técnica quirúrgica, novedosa en el medio nacional, de abordaje vaginal, para el tratamiento del prolapso apical: la suspensión transvaginal alta a ligamentos úterosacros (STALUS). Método: Estudio descriptivo longitudinal, de 57 pacientes con defectos apicales, a los cuales se les realizó esa técnica entre Diciembre de 2002 y Octubre 2005. Se realizó estadística descriptiva y test t (2 muestras) para análisis de pronóstico anatómico (POP-Q). Para análisis de potenciales factores pronósticos se utilizó ANOVA, regresión lineal y logística. Resultados: El tiempo operatorio promedio fue de 151 minutos. El resultado anatómico (POP-Q), pre y postoperatorio, resultó favorable y estadísticamente significativo, en los nueve puntos evaluados, 49 de las 54 pacientes fueron seguidas en promedio durante 15 meses. En el compartimiento apical (punto C) obtuvimos curación del $89 \%$ y no hubo fracasos. En la pared anterior, $22 \%$ de las pacientes recidivaron. En cuanto a las complicaciones, se produjo una fístula ureterovaginal. Conclusiones: Tomando las precauciones necesarias, es una técnica segura y reproducible, con buenas tasa de curación. Asegurar la indemnidad del uréter, siempre será una obligación. La recidiva en pared anterior, aunque sea asintomática, resulta ser extremadamente alta, lo que nos obliga a pensar en nuevas técnicas de abordaje de este compartimiento.

\section{PALABRAS CLAVE: Prolapso genital, prolapso apical, defecto apical, abordaje vaginal, ligamentos úterosacros}

\section{SUMMARY}

Objective: To describe a novel surgery technique in the national ground, of vaginal approach for the treatment of apical prolapse: the transvaginal high suspension to the uterosacral ligaments (STALUS). Method: It is a longitudinal descriptive study that included 57 patients with apical support defects, in which this technique was performed between December 2002 and October 2005. Descriptive statistics and t test were performed for the anatomical outcome (POP-Q). For the potential prognosis factors, ANOVA, lineal regression 
and logistic, were used. Results: The average surgery time was 151 minutes. The anatomical result (POP$Q$ ), before and after surgery, was favourable and significant in the nine points evaluated. 49 of 54 patients were followed for 15 months in average. In the apical compartment (C point) we got an 89\% of cure and there were no failure. In the anterior wall, instead, $22 \%$ of our patients recurred. About complications, there was an ureterovaginal fistula. Conclusions: If all precautions are taking, there is a secure and reproducible technique, with good cure rate. To secure the ureter it is always an obligation. The recurrence in the anterior wall, even been asymptomatic, is too high, that make us think in new techniques in order to manage this compartment.

\section{KEY WORDS: Genital prolapse, apical prolapse, apical defect, vaginal approach, uterosacral ligaments}

\section{INTRODUCCIÓN}

El prolapso genital es una patología frecuente, que en un número no despreciable de casos requerirá de una intervención quirúrgica. Estudios en población cerrada, revelan que la probabilidad de una mujer de ser operada a lo largo de su vida, de una disfunción del piso pélvico, alcanza a $11 \%$ (1). Los defectos apicales incluyen al histerocele, al prolapso de cúpula vaginal y al enterocele apical. Según los postulados de DeLancey (2), tener una adecuada suspensión del compartimiento apical o nivel I, es crítico para el soporte del resto del canal vaginal. El abordaje quirúrgico puede ser abdominal, vaginal o laparoscópico, existiendo más de 40 procedimientos descritos en la literatura. El objetivo general de este estudio es describir una técnica quirúrgica, novedosa en el medio nacional, de abordaje vaginal, para el tratamiento del prolapso apical, como es la suspensión transvaginal alta a ligamentos úterosacros, descrita por Shull y cols en 2000 (3). Los objetivos específicos son describir el pronóstico anatómico de la intervención, utilizando la sistema de cuantificación POP-Q (4) y evaluar la morbilidad y posibles factores pronósticos.

\section{MATERIAL Y MÉTODO}

Estudio descriptivo longitudinal, que incorporó 57 pacientes con defectos apicales, a los cuales se les realizó la técnica STALUS (3) entre Diciembre de 2002 y Octubre 2005, en dos centros diferentes. Los criterios de inclusión fueron pacientes con defectos apicales primarios (no recidivados), con deseo de conservar vagina funcional y sin contraindicación de cirugía vaginal. La cuantificación de la severidad del prolapso se realizó mediante la aplicación del sistema POP-Q (4).

Descripción de la técnica quirúrgica: Se realiza histerectomía vaginal en casos de histerocele. En prolapso de cúpula o enterocele apical, se entra a cavidad peritoneal con incisión vertical en mucosa vaginal a nivel del apex. Una vez abierto el peritoneo, se coloca packing intrabdominal para rechazar las vísceras y se ubica la porción más alta (distal) de los ligamentos útero sacros, mediante visualización, palpación y tracción de su porción proximal. El segmento útil se localiza posterior y medial a la espinas ciáticas en la hora 4 y 8 , para evitar el uréter. Solo cuando se está seguro de la toma, se pasan dos suturas de prolene en cada ligamento. Los extremos de estas suturas se pasan, a nivel anterior, por la fascia pubocervical y a nivel posterior, por la fascia rectovaginal. Con esta maniobra, se reconstituye, en forma anatómica, el nivel I de DeLancey. Cualquier otro defecto detectado, se corrige en forma contemporánea, mediante reparación sitio-específica, ya sea anterior o posterior. Luego se realiza cistoscopia intraoperatoria, previa inyección de $5 \mathrm{ml}$ de índigo carmín endovenoso, para asegurar indemnidad ureteral. La visualización de flujo azul, tipo chorro, por ambos meatos ureterales, mientras se traccionan la suturas de prolene, asegura la indemnidad de ambos uréteres. Solo se procede a anudar los puntos de anclaje, para ascender la cúpula vaginal, una vez que el paso anterior se ha cumplido en forma estricta. El pronóstico anatómico postoperatorio, se realizó mediante examen físico realizado por alguno de los autores, aplicando sistema de cuantificación POP-Q, tal cual lo describen sus creadores (4).

Análisis estadístico: Se realizó estadística descriptiva y t test (2 muestras) para análisis de pronóstico anatómico (POP-Q). Para análisis de potenciales factores pronósticos se utilizó Anova, regresión lineal (variables continuas) y logística (variables categóricas) en programa estadístico STATA 7.0. Se consideró significativo un $p<0,05$. Se utilizaron los criterios pronósticos recomendados por el NIH en consenso del año 1999 (5), en los cuales se definen tres categorías: curación, mejoría y recurrencia o fracaso (Tabla I). 
Tabla I CRITERIOS PRONÓSTICOS

Pronóstico
1. Anatómico óptimo (curación):
. Sin evidencia de descenso de cúpula, estadío 0.
. Punto C localizado entre -LVT y -(LVT -2) cm.

2. Anatómico satisfactorio (mejoría):

Descenso de cúpula hasta $1 \mathrm{~cm}$ sobre himen, estadío 1.

- Punto C entre -1 y LVT $-2 \mathrm{~cm}$.

3. Anatómico insatisfactorio o fracaso (recurrencia):

- Descenso de cúpula menos de $1 \mathrm{~cm}$ del hímen o más (estadio II, sin cambios o empeoramiento estadio preoperatorio)

LVT: largo vaginal total.

Fuente: Weber AM, Abrams P, Brubaker L, Cundiff G, Davis $G$, Dmochowski RR, et al. The standardization of terminology for researchers in female pelvic floor disorders. Int Urogynecol J Pelvic Floor Dysfunct 2001;12:178-86.

\section{RESULTADOS}

Se reclutaron 57 pacientes que cumplían con los criterios de inclusión. En tres de ellas, no fue posible realizar la cirugía completa, dos por un síndrome adherencial pelviano severo y la otra por la formación de hematoma retroperitoneal. De las 54 pacientes, tenemos 5 con seguimiento incompleto $(9,2 \%)$, que se consideraron perdidas, por lo que se detalla pronóstico de las 49 restantes. En la Tabla II se muestran las características de la cohorte en estudio, destacando una edad promedio de 59 años, con $83 \%$ postmenopáusicas, $50 \%$ con sobrepeso u obesidad, $50 \%$ constipadas y $35 \%$ con cirugía pelviana previa, incluyendo histerectomía, procedimientos para incontinencia urinaria y reparaciones anteriores y posteriores de prolapso genital. En la Tabla III se detallan las características de la población intervenida. Con respecto al tipo y severidad del prolapso, destaca un $57 \%$ de pacientes con estadíos III o IV y $22 \%$ de prolapso de cúpula; $74 \%$ tenía incontinencia urinaria de esfuerzo, clínica u oculta.

En todas las pacientes hubo procedimientos quirúrgicos asociados, incluyendo colporrafias anteriores, reparaciones posterior sitio específico, histerectomía vaginal y cintas suburetrales libres de
Tabla II CARACTERÍSTICAS DE LAS PACIENTES

\begin{tabular}{lc}
\hline Variables & $\mathrm{n}=54$ \\
\hline Edad (años \pm DE; rango) & $59 \pm 9 ; 40-81$ \\
Partos vaginales ( \pm DE) & $2,9 \pm 1,5$ \\
$\begin{array}{l}\text { Recién nacido de mayor } \\
\text { peso (gramos } \pm \text { DE) }\end{array}$ & $3.662 \pm 445$ \\
Menopáusicas (\%) & $27(50)$ \\
IMC >85 (\%) & $24(46)$ \\
Constipación crónica (\%) & $18(35)$ \\
Cirugía pélvica previa (\%)
\end{tabular}

DE: desvío estándar. IMC: índice de masa corporal.

tensión (TVT, IVS anterior y TVT-O), el detalle se muestra en Tabla IV.

El tiempo operatorio promedio, incluyendo todos los tiempos quirúrgicos, fue de $151 \pm 35$ minutos (rango: 90-240 minutos). El resultado anatómico, expresado en sistema de cuantificación POP-Q, pre y postoperatorio, se detalla en Tabla $V$, siendo favorable y estadísticamente significativo, en los nueve puntos evaluados. El largo vaginal total disminuyó de 9 a $8,3 \mathrm{~cm}$, lo que fue significativo. Fueron seguidas 49 de las 54 pacientes, en promedio durante 15 meses. En el compartimiento apical (punto C) obtuvimos curación en 44 de 49 pacientes (89\%) y no hubo fracasos. En la pared anterior, 11 de 49 (22\%) pacientes recidivaron, las cuales fueron asintomáticas. Durante el período de observación, no hubo reoperaciones por recurrencias (Tabla $\mathrm{VI}$ ).

En cuanto a las complicaciones, se produjo una fístula ureterovaginal, que se consideró como mayor. Esta requirió un neoimplante ureteral a los 14 días de operada. Las otras, se consideraron menores e incluyen vagina corta en 2 pacientes y prolene palpable en 9.

En el análisis de factores pronósticos (regresión lineal y logística), solo el prolapso de cúpula y el antecedente de cirugía pélvica, se asociaron a un peor pronóstico anatómico en el compartimiento apical $(p<0,05)$. En pared anterior, ninguno de los factores estudiados se asoció a un mal resultado anatómico.

\section{DISCUSIÓN}

Se trata de una experiencia con una técnica 
Tabla III

CARACTERÍSTICAS CLÍNICAS DE LA POBLACIÓN INTERVENIDA

\begin{tabular}{lll}
\hline Característica clínica & & $\mathrm{n}(\%)$ \\
\hline Estadío preoperatorio & II & $21 / 49(43)$ \\
& III & $19 / 49(39)$ \\
& IV & $9 / 49(18)$ \\
Incontinencia de orina de esfuerzo & Sin incontinencia & $13 / 50(26)$ \\
& Incontinencia clínica & $25 / 50(50)$ \\
& Incontinencia oculta & $12 / 50(24)$ \\
Tipo de prolapso & Cúpula & $11 / 52(22)$ \\
& Histerocele & $41 / 52(78)$ \\
\hline
\end{tabular}

Tabla IV CIRUGÍAS ASOCIADAS

\begin{tabular}{lc}
\hline Tipo de cirugía & $\mathrm{n}(\%)$ \\
\hline Cirugías asociadas & $54 / 54(100)$ \\
Histerectomía vaginal & $42 / 54(78)$ \\
Reparación anterior y posterior & $52 / 54(96)$ \\
Sling suburetral & $36 / 54(70)$ \\
Esfinteroplastía & $1 / 54(1,9)$ \\
\hline
\end{tabular}

quirúrgica novedosa en el medio nacional con resultado anatómico óptimo en la gran mayoría las pacientes (89\%). Nuestros resultados son comparables con las pocas series que existen publicadas en la literatura internacional, las cuales muestran tasas de éxito que van del 81 al 100\% (3,6-10). Tomando las precauciones necesarias, es una técnica segura y reproducible. Asegurar la indemnidad del uréter, siempre será una obligación, para ello es necesario tener claro los conceptos anatómicos del piso pélvico y del trayecto del uréter pelviano, junto a un entrenamiento guiado en la técnica quirúrgica, ambos como prevención primaria. Es indispensable, como prevención secundaria, realizar la cistoscopia intraoperatoria y visualizar el chorro azul de índigo carmín, por ambos meatos ureterales, pre- viamente inyectado vía endovenosa. Esta técnica tiene una sensibilidad de $94,4 \%$ y una especificidad de $99,5 \%$ con $0,4 \%$ de falsos positivos y $0,3 \%$ de falsos negativos (11). La complicación mayor en nuestro estudio se debió a una fístula ureterovaginal, en donde el análisis de nuestro grupo en la explicación de su formación, habría radicado en que en esa paciente, si bien se visualizó el chorro azul por el meato ureteral, este fue débil, fino y tardío (más de 12 minutos), lo que constituye un falso negativo de la técnica.

La recidiva en pared anterior, aunque sea asintomática, es extremadamente alta. Corresponde con cifras reportadas internacionalmente (hasta $40 \%$ ). Esta alta tasa de recurrencia hace indispensable el desarrollo de nuevas técnicas que permitan disminuir de manera eficaz y segura la reintervención quirúrgica de estas pacientes. Lamentablemente, no pudimos identificar, en nuestro grupo de estudio, factores predictores de este mal resultado.

Es imprescindible manejar alternativas quirúrgicas para casos en que la técnica no sea factible, como en los casos expuestos en nuestro trabajo, como el síndrome adherencial que haga impracticable el abordaje del ligamento útero-sacro en su porción alta. Algunas alternativas son la fijación al ligamento sacrociático, ligamento úterosacro extraperitoneal y prolift posterior.

Nuestro trabajo confirma la utilidad del sistema de cuantificación POP-Q, en análisis del pronóstico anatómico de la reparación del prolapso, ya que resulta ser cuantitativo, objetivo, y permite realizar un 
Tabla V

CUANTIFICACIÓN POP-Q PRE Y POS OPERATORIO

\begin{tabular}{lccc}
\hline POP-Q $^{*}$ & Pre operatorio $(\mathrm{cm})$ & Pos operatorio $(\mathrm{cm})$ & Valor $\mathrm{p}$ \\
\hline $\mathrm{Xa}$ & $\mathrm{X} \pm \mathrm{DE}$ & $\mathrm{X} \pm \mathrm{DE}$ & $<0,001$ \\
$\mathrm{Ba}$ & $+0,3 \pm 2,0$ & $-2,8 \pm 0,5$ & $<0,001$ \\
$\mathrm{C}$ & $+1,4 \pm 3,0$ & $-1,9 \pm 1,1$ & $<0,001$ \\
$\mathrm{Ap}$ & $-0,7 \pm 1,9$ & $-7,3 \pm 2,0$ & $<0,001$ \\
$\mathrm{Bp}$ & $0 \pm 2,8$ & $-2,8 \pm 0,4$ & $<0,001$ \\
$\mathrm{LVT}$ & $9,0 \pm 1,3$ & $-2,8 \pm 0,4$ & $<0,002$ \\
$\mathrm{Hg}$ & $3,9 \pm 0,9$ & $8,3 \pm 1,4$ & $<0,001$ \\
$\mathrm{Cp}$ & $2,6 \pm 0,9$ & $3,1 \pm 1,4$ & $<0,001$ \\
$\mathrm{D}$ & $-3,8 \pm 5,4$ & $3,2 \pm 0,8$ & - \\
\hline
\end{tabular}

$\mathrm{X} \pm \mathrm{DE}$ : promedio \pm desvío estándar.

POP-Q: pelvic organ prolapse cuantification system. Aa - Ba: pared vaginal anterior. C: cérvix o cúpula. Ap - Bp: pared vaginal posterior. LVT: largo vaginal total. Hg: hiato genital. Cp: cuerpo perineal. D: fondo de saco de Douglas.

${ }^{*}$ Fuente: Bump RC, Mattiasson A, Bo K, Brubaker LP, DeLancey JO, Klarskov P, et al. The standardization of terminology of female pelvic organ prolapse and pelvic floor dysfunction. Am J Obstet Gynecol 1996;175:10-7.

\section{Tabla VI \\ RESULTADOS FINALES}

\begin{tabular}{|c|c|}
\hline Variable & Resultado \\
\hline Seguimiento en meses ( $X \pm D E ;$ rango) & $15 \pm 9 ; 3-36$ \\
\hline Pérdida de seguimiento $\mathrm{n}(\%)$ & $5 / 54(9,2)$ \\
\hline Compartimento apical (punto C) & $\mathrm{n}=49$ \\
\hline - curación n (\%) & $44 / 49(89)$ \\
\hline - mejoría n (\%) & $5 / 49(11)$ \\
\hline - insatisfactorio n (\%) & $0 / 49(0)$ \\
\hline Compartimento anterior (Aa y $\mathrm{Ba}$ ) & $\mathrm{n}=49$ \\
\hline - curación mas mejoría n (\%) & $38 / 49(78)$ \\
\hline - insatisfactorio n (\%) & $11 / 49(22)$ \\
\hline - reoperación por recurrencia n (\%) & $0 / 49(0)$ \\
\hline
\end{tabular}

$\mathrm{X} \pm \mathrm{DE}$ : promedio \pm desvío estándar. análisis estadístico más rico en la comparación pre y post operatoria.

Nuestras conclusiones están limitadas por el seguimiento insuficiente (15 meses) y por el diseño descriptivo, sin embargo, este siempre es un primer paso para el desarrollo de trabajos prospectivos idealmente randomizados, ya que muestra la seguridad y factibilidad de la técnica, con resultados comparables con lo publicado en la literatura internacional.

\section{CONCLUSIÓN}

La suspensión transvaginal alta a ligamentos úterosacros, es una alternativa eficaz, factible y segura, para el manejo de los defectos apicales primarios.

\section{BIBLIOGRAFÍA}

1. Olsen AL, Smith VJ, Bergstrom JO, Colling JC, Clark AL. Epidemiology of surgically managed pelvic organ prolapse and urinary incontinence. Obstet Gynecol 1997;89:501-6.

2. DeLancey JOL. Anatomic aspects of vaginal eversion after hysterectomy. Am J Obstet Gynecol 1992;166(6 Pt 1):1717-24

3. Shull BL, Bachofen C, Coates KW, Kuehl TJ. A trans- 
vaginal approach to repair of apical and other associated sites of pelvic organ prolapse with uterosacral ligaments. Am J Obstet Gynecol 2000;183:1365-74.

4. Bump RC, Mattiasson A, Bo K, Brubaker LP, DeLancey JO, Klarskov $P$, et al. The standardization of terminology of female pelvic organ prolapse and pelvic floor dysfunction. Am J Obstet Gynecol 1996;175:10-7.

5. Weber AM, Abrams P, Brubaker L, Cundiff G, Davis G, Dmochowski RR, et al. The standardization of terminology for researchers in female pelvic floor disorders. Int Urogynecol J Pelvic Floor Dysfunct 2001;12:17886.

6. Jenkins VR. Uterosacral ligament fixation for vaginal vault suspension in uterine and vaginal vault prolapse. Am J Obstet Gynecol 1997;177:1337-43.

7. Barber M, Visco AG, Weidner AC, Amundsen CL, et al. Bilateral uterosacral ligament vaginal vault suspension with site-specific endopelvic fascia defect repair for treatment of pelvic organ prolapse. Am J Obstet Gynecol 2000;183:1402-11.
8. Karram M, Goldwasser S, Kleeman S, Steele A, Vassallo $B$, Walsh $P$. High uterosacral vaginal vault suspension with fascial reconstruction for vaginal repair of enterocele and vaginal vault prolapse. Am J Obstet Gynecol 2001; 185: 1339-42.

9. Amundsen C, Flynn BJ, Webster GD. Anatomical correction of vaginal vault prolapse by uterosacral ligament fixation in women who also require a pubovaginal sling. J Urol 2003;169:1770-4.

10. Silva WA, Pauls RN, Segal JL, Rooney CM, Kleeman SD, Karram MM. Uterosacral ligament vault suspension: five-year outcomes. Obstet Gynecol 2006;108:255-63.

11. Gustilo-Ashby AM, Jelovsek JE, Barber MD, Yoo EH, Paraiso MF, Walters MD. The incidence of ureteral obstruction and the value of intraoperative cystoscopy during vaginal surgery for pelvic organ prolapse. Am J Obstet Gynecol 2006;194:1478-85. 